\title{
EXPERIENCIAS Y APRENDIZAJES DEL DESARROLLO DE VARIEDADES DE FRIJOL DE MANERA PARTICIPATIVA EN EL NORTE DE NICARAGUA1
}

\author{
Conny Almekinders ${ }^{2}$, Julio Molina-Centeno ${ }^{3}$, Rolando Herrera-Torrez ${ }^{4}$, Santos Luis Merlo-Olivera ${ }^{5}$, \\ José Manuel González-Suárez, Juan García-Carrasco ${ }^{5}$
}

\begin{abstract}
RESUMEN
Experiencias y aprendizajes del desarrollo de variedades de frijol de manera participativa en el norte de Nicaragua. Esta publicación describe un proyecto piloto de fitomejoramiento en el Norte de Nicaragua. Semillas de frijol de la generación F3 con base en tres cruces triples, hechos en CIAT, Colombia, fueron entregadas a cinco agricultores de dos localidades. Por medio de selección dentro y entre familias, cada agricultor desarrolló una línea avanzada de frijol con resistencia al Mosaico Dorado adaptado a sus condiciones de producción y preferencias. La comparación y validación de las líneas avanzadas con una variedad testigo, resultó en la identificación de dos materiales que se comportaron mejor que el testigo en el área, considerando las evaluaciones de rendimiento, valor comercial (color de grano) y características culinarias. Estas líneas promisorias fueron bautizadas y lanzadas en octubre 2004. El proceso de participación es descrito considerando un número de factores incluyendo el proceso en el transcurso de tiempo. Los resultados de este proyecto piloto prueban que es posible desarrollar variedades superiores en campos de agricultores y con la participación de ellos. El desafío del escalamiento e institucionalización presentan preguntas que solamente se puede contestar con experiencias reales.
\end{abstract}

Palabras claves: Fitomejoramiento participativo, $P$ haseolus vulgaris L., experimentación campesina, agricultura de bajos insumos.

\begin{abstract}
Learning experiences obtained from the development of bean varieties through participative breeding in Northern Nicaragua. This publication describes a pilot project of Participatory Plant Breeding in Northern Nicaragua. F3 bean families derived from three triple crosses made in CIAT, Colombia, were given to five farmers in two localities. Using within- and between-family selection, each farmer developed an advanced line resistant to Bean Mosaic Virus and adapted to his own production conditions and preferences. Comparison and validation of these advanced lines with local test variety resulted in the identification of two promising lines that outperformed the local improved variety considering yield, commercial (seed color) and culinary characteristics. These lines were named and released as varieties in October 2004. This process of participatory research is described considering a range of factors influencing the process over time. The results of this pilot process prove that it is possible to develop superior varieties in farmers' fields utilizing farmers' expertise. The challenges of up-scaling and institutionalization of PPB are raising questions that can only be answered through real life experiences'.
\end{abstract}

Key words: Participatory plant breeding, Phaseolus vulgaris L., participatory on-farm research, low-input agriculture.

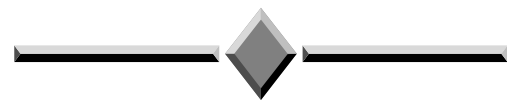

\footnotetext{
1 Recibido: 16 de enero, 2006. Aceptado: 20 de julio, 2006.

2 Departamento de Tecnología y Desarrollo Agrario, Universidad de Wageningen, Holanda. Correo electrónico: c_almekinders@zonnet.nl

3 Instituto Nicaragüense de Tecnología Agropecuaria (INTA), Estelí Nicaragua.

4 Centro para la Investigación, la Promoción y el Desarrollo Rural y Social (CIPRES). Pueblo Nuevo, Nicaragua. Correo electrónico: fmcipres@ibw.com.ni

5 Agricultor-fitomejorador El Rosario, Pueblo Nuevo, Nicaragua.
} 


\section{INTRODUCCIÓN}

El concepto de fitomejoramiento participativo (FP) surgió como respuesta a las críticas frente al impacto que ha tenido el fitomejoramiento convencional (FC) en la producción agrícola en campos de pequeños agricultores (Hardon 1995; Almekinders y Elings 2001). El FC es considerado exitoso en generar variedades con mayor productividad. Sin embargo, la expresión de este mayor potencial es restringido en campos con niveles reducidos de insumos y bajo condiciones de estreses complejos. Además, las preferencias de los pequeños agricultores en muchos casos resultan ser variables, y distintas a las de los productores que únicamente cultivan para la comercialización. Los pequeños agricultores por ejemplo valorizan más el rendimiento en años con condiciones extremas, es decir cuando se presenta sequía o alta presión de enfermedades o plagas, y la calidad culinaria. En general, los programas de fitomejoramiento de carácter centralizado y enfocado en una agricultura de altos niveles de insumos, a menudo no generan variedades que satisfacen las necesidades de los pequeños agricultores. Como consecuencia, las variedades de los programas de FC han tenido un impacto menor en la producción de la agricultura de pequeña escala.

Con el FP se pretende dar solución a algunas de las restricciones con la que cuenta el FC. Entonces, se le puede considerar una estrategia complementaria al FC, dirigiéndose en primer instante a áreas variables y marginales, o a pequeños productores que cultivan autoconsumo y el mercado. La promoción de FP se basa también en el argumento cita a los productores como los fitomejoradores desde el inicio de la agricultura, hasta que el fitomejoramiento se desarrolló como una especialidad científica. Entonces, sus capacidades de experimentación y selección serán significativas. Sin embargo, el fitomejoramiento, con el descubrimiento de los genes, se desarrolló como una ciencia aplicada de la genética con prácticas y conocimientos más alejados a la producción agrícola. La idea de re-involucrar a los agricultores en una manera directa (Hardon 1995; Berg 1996) da lugar a preguntar si los agricultores están en posición de tomar un papel en el desarrollo de variedades que resultará en variedades de mayor potencial en sus campos. En un trabajo experimental con frijol en Colombia se mostró que los agricultores estaban en capacidad de seleccionar materiales segregantes de frijol, así desarrollaron materiales de similar rendimiento que los materiales seleccionados por fitomejoradores (Kornegay et al. (1996). Pero, este trabajo no reflexionó sobre el papel de los actores participantes. ¿Cuál podría ser el papel de agricultores y otros en FP y en cuáles condiciones se les deberá tomar en cuenta? ¿Cuáles serán las implicaciones para los programas de fitomejoramiento y la difusión de semilla? Y, ¿hasta dónde utilizar los escasos recursos de FC y entonces dañara los programas que se necesitan para desarrollar variedades que ayudan a alimentar a la población urbana? Así, existe una serie de preguntas que únicamente se puede contestar con evidencias de una experiencia real.

Esta publicación presenta las experiencias de un proyecto piloto en Nicaragua que fue implementado con un grupo de 50 agricultores, una ONG y un fitomejorador del Instituto Nicaragüense de Tecnología Agropecuaria (INTA) (Almekinders et al. 2002). En el proyecto se abarcaban trabajos con maíz y frijol. Esta publicación se enfoca en las experiencias con frijol, documenta la metodología usada y los resultados, es decir las experiencias y los materiales producidos. Tiene como objetivo también compartir las consideraciones que jugaron un papel en las decisiones que se tomaron en el transcurso del proceso. Basado en las experiencias, se revisan las suposiciones del concepto de FP y se discute las implicaciones para programas de fitomejoramiento.

\section{MATERIALES Y MÉTODOS}

El proyecto tiene su raíz en una iniciativa de formular un programa regional de Fitomejoramiento Participativo, para explorar las oportunidades que el concepto de FP tendrá en combinar la generación de diversidad genética con el alivio de la pobreza de los agricultores, a lo cual muchos países se comprometieron en el Convenio de Biodiversidad (CBD 1992). El objetivo fue desarrollar y comparar metodologías de FP. El proyecto piloto en Pueblo Nuevo y Condega, Nicaragua, forma un caso en el contexto de un programa regional que también involucra experiencias en otros países mesoamericanos (véase el volumen 17(3) 2006 de la Revista Agronomía Mesoamericana).

En un proceso de consulta y formulación del proyecto, el Centro para la Promoción, la Investigación y 
el Desarrollo Rural y Social (CIPRES) expresó su interés en un proyecto acerca de FP. CIPRES es una ONG con sede principal en Managua y con oficinas en Somoto, Pueblo Nuevo y Chinandega. CIPRES tenía amplia experiencia con el tema de semillas, por lo que implementó proyectos de producción artesanal de semilla (PAS) y considera el tema semillas como un componente esencial en la seguridad alimenticia.

En diciembre del año 1999, CIPRES convocó a una reunión en Pueblo Nuevo, a la que asistieron 50 agricultores interesados en desarrollar variedades de frijol y maíz, los dos cultivos en los cuales se enfocaba el programa regional de FP. Se explicó a los agricultores que no existía una metodología probada y no se podría garantizar éxito. Lo único que se podría manifestar con seguridad es que su participación requeriría un compromiso de largo plazo, es decir de varias campañas. El proyecto no iba a disponer de recursos para pagar a los agricultores por sus esfuerzos. Aparentemente, el deseo de los agricultores de tener variedades adaptadas a sus condiciones era fuerte. El Mosaico Dorado (MD), transmitido por la mosca blanca limitó seriamente la siembra de frijol en la localidad. Al inicio del proyecto, la única variedad que tenía tolerancia fue DOR 364, un frijol de un color rojo oscuro poco apreciado en Nicaragua, lo que causó un precio bajo en el comercio por intermediarios. En la parte de la localidad donde la presión del MD estaba más fuerte, los agricultores prácticamente no sembraron frijol y muchos se vieron obligados a comprar frijol para su consumo. Esta situación, combinado con la confianza que tenían en la organización CIPRES, les hizo comprometerse.

Como CIPRES y los agricultores no tenían experiencia o conocimiento específico de fitomejoramiento, buscaron el apoyo del fitomejorador de INTA-Estelí, la estación experimental del INTA más cercana a Pueblo Nuevo. Por medio de un memorando de entendimiento entre CIPRES y INTA, se facilitó la participación del fitomejorador, es como una actividad complementaria en su programa de tareas. CIPRES se comprometió a pagar gastos de transporte del fitomejorador.

El proyecto piloto consistó en una serie de ensayos de selección y evaluación. Como material de selección se emplearon materiales disponibles en este momento: 15 familias generadas en el CIAT, Colombia, de tres cruzamientos triples, que incluyó a Tío
Canela 75 (Cuadro 1). El material fue recibido por el fitomejorador de INTA-Estelí en las generaciones F3 y F4. Se sembró parte de las semillas en la Estación Experimental de Estelí, y se proporcionó semillas de las mismas familias a los agricultores que estaban dispuestos en sembrar un ensayo en sus campos.

En una reunión de planificación, después de explicaciones de parte del fitomejorador sobre la implementación de los ensayos, el grupo de agricultores en consulta con el fitomejorador y el técnico de CIPRES - decidieron establecer ensayos de evaluación y selección en campos de cinco agricultores. Se tomó en cuenta que los cinco agricultores tenían en sus campos condiciones diferentes, tal que la variación de las condiciones de las comunidades estuvieran representadas (Cuadro 1). Los cinco agricultores (desde aquí indicados como agricultores-fitomejoradores) se encargaron individualmente de sus propias evaluaciones y selecciones. El manejo fue dejado a criterio del agricultor, con la condición que se aplicara un mínimo de fertilizantes y que se coordinara las épocas de riego para garantizar la selección de materiales adaptadas a baja fertilidad y condiciones locales de humedad o sequía. En cada campaña, el grupo de 50 agricultores se reunió una o dos veces para observar las parcelas, además de la época de cosecha, y para la reflexión y planificación de la siguiente siembra. Aunque los cinco

Cuadro 1. Genealogía de las familias de frijol, agricultores-fitomejoradores y características de cinco localidades donde se ubicaron los ensayos de selección, comparación y validación. Nicaragua, 2005.

\section{Genealogía (No. familias por cruce)}

(MAM 38 x DICTA 17) F1 x Tío Canela (1 familia)

Tío Canela 75 x (Catrachita x De Celaya) F1 x (FEB 212 x VAX 6) F1)F1 (4 familias)

(VAX 3 x Catrachita) F1 x Tío Canela 75 (10 familias)

\section{Agricultores y detalles de localidades}

\begin{tabular}{llcc}
\hline \multicolumn{1}{c}{ Agricultor } & Localidad & $\begin{array}{c}\text { Altura } \\
\text { (msnm) }\end{array}$ & $\begin{array}{c}\text { Precipitación } \\
\text { (mm por año) }\end{array}$ \\
\hline Juan García & Santa Rosa & 850 & 750 \\
José M. González & Paso Hondo & 630 & 600 \\
Pedro Gómez & La Lima & 1000 & 1000 \\
Santos L. Merlo & El Rosario & 650 & 600 \\
Jairo Videa & Río Abajo & 600 & 650 \\
\hline
\end{tabular}


agricultores tenían la decisión final sobre las selecciones, estos se apoyaron en el criterio del grupo. Entonces, se puede considerar que el proyecto piloto se desarrolló como cinco procesos de selección de manera paralela.

\section{RESULTADOS Y DISCUSIÓN}

\section{La participación y esquema de evaluación y selección}

Mientras que los cinco agricultores-fitomejoradores manejaron el cultivo según su criterio y tomaron las decisiones finales sobre cuales plantas o familias seleccionar, el esquema de selección fue discutido y decidido en grupo. Como los agricultores no tenían experiencia en selección de materiales segregantes y su desarrollo hacia variedades, la asesoría del fitomejorador en estas planificaciones fue crucial. El fitomejorador en la primera fase del proyecto tomó un papel que se puede definir como profesor-experto. El explicaba elementos claves de la genética y proporcionaba argumentos y consideraciones que permitieron a los agricultores tomar 'decisiones informadas'. Es difícil denominar el carácter de participación de los diferentes actores en este fase con base en el poder de decisión (Sperling et al. 2001). En la realidad el grupo de productores con el técnico y el fitomejorador 'en conjunto' se pusieran de acuerdo, tomando en cuenta opiniones de cada uno. Algunos referirían a este tipo de participación como 'decisiones negociadas'. Sin embargo, nosotros sentimos que éste no refleja la relación que existía en esta fase del proyecto. Si que había puntos de diferentes opiniones, pero es más justo definirlos como puntos de dudas, pues no existían protocolos o experiencias anteriores y entonces no se sabía cual opción (método, estrategia, etc.) resultara más efectiva. Entonces, consideramos que la mejor definición de este tipo de participación podría ser decisión consensuada con base en la mejor información.

El esquema que cada uno de los cinco agricultores-fitomejoradores siguieron con pequeñas variaciones, fue el siguiente:

2000 Primera: cada uno de los cinco agricultores-fitomejoradores sembraron aproximadamente 520 semillas (cuatro surcos de $5 \mathrm{~m}$ ) de cada una de las 15 familias F3-F4. El diseño de los experimentos fue sugerido por el fitomejorador del INTA, el técnico de CIPRES ayudó en la siembra. Los agricultores seleccionaron de cada progenie hasta 20 plantas, resultando en 300 familias F4-F5 para la siguiente siembra.

Postrera: 300 familias F4-F5 (surcos de 30 metros) => selección de aprox. 80 familias.

2001 Primera: 80 familias F5-F6 sembradas $=>$ mejores 30 familias seleccionadas

Postrera: 30 familias F6-F7 sembradas $=>$ aprox. siete mejores familias seleccionadas Riego: cinco líneas F7-F8 y un testigo (DOR 364) sembrados en campo de un agricultor (S.L. Merlo). Los cinco agricultores proporcionaron su mejor línea seleccionada, para esta evaluación comparativa. El ensayo sirvió también para multiplicación de la semilla.

2002 Primera: cinco líneas seleccionadas (F8-F9) sembradas en campos de cuatro agricultores => selección de las dos mejores líneas.

Postrera: 26 ensayos con las cinco mejores líneas seleccionadas por los cinco agricultores, sembradas en finca en los alrededores de las dos comunidades. La variedad INTA-Masatepe fue incluída en los ensayos como variedad testigo. Las cinco líneas seleccionadas y el control fueron evaluados por los agricultores por su rendimiento y calidad culinaria.

2003 Primera: 22 ensayos adicionales sembrados con las dos mejores selecciones de la postrera anterior e INTA Masatepe como testigo. Esto completa la colección de datos de un total de los 48 ensayos de evaluación. Los análisis estadísticos de los datos identificaron a las dos mejores líneas.

Postrera: Las dos líneas identificadas fueron nombradas JM 12-7 y Santa Elena. Se multiplicó semilla de estas líneas.

2004 Octubre: 'liberación de dos variedades para uso comercial (no inscritas en el registro oficial de variedades).

\section{Criterios de selección}

Para los agricultores-fitomejoradores los criterios más importantes en la evaluación y selección fueron: 
resistencia al Mosaico Dorado (MD), color y rendimiento. La incidencia del Mosaico Dorado en la zona fue muy fuerte (presencia de cultivos hospederos como tabaco y tomate, y el vector mosca blanca con resistencia a los agroquímicos) e hizo imposible la producción de frijol en zonas bajas.

La selección por resistencia al MD fue relativamente fácil de observar y determinar la resistencia en una escala de uno a nueve, que no resultó complicada para los agricultores. Los agricultores seleccionaron plantas individuales de cada familia en la cosecha de primera 2000. Cuando las plantas de la familia mostraron poca tolerancia, se eliminó la familia completa. Así, dependiendo del agricultor, se seleccionaron 200300 plantas, originadas de dos a seis familias. Después se seleccionó por familia. Al final, las cinco líneas promisorias que fueron identificadas por los cinco agricultores como las mejores se originaron del cruce (VAX 3 x Catrachita) F1 x Tío Canela 75.

La tolerancia a la sequía fue también una característica importante, que contribuye al rendimiento. Hubo agricultores que usaron sus criterios personales. Varió la preferencia para el tipo de planta, periodo floración (corto o prolongado), tamaño de vainas, tamaño y color de grano. Uno de los cinco agricultores prestaba atención al llenado de la vaina, por lo que seleccionó vainas más cortas que también estaban llenas en condiciones de estrés. Otro agricultor decidió aplicar un riego fuerte (aspersión) porque consideraba que muchas veces después de un periodo de sequía son los chaparrones fuertes los que hacen volcar las plantas. Entonces después del riego marcó las plantas que se quedaron en pie, resistentes al acame.

La calidad culinaria se obtuvo en la fase de evaluación de las mejores líneas. No había suficiente semilla para pruebas en la fase anterior. Entonces, introdujeron solamente los cinco mejores materiales seleccionados por los cinco agricultores-fitomejoradores. En la prueba culinaria se consideraron el tiempo necesario para cocinar el frijol y la consistencia en la sopa. Sobresalió el JM-12.7 por su buena calidad en la sopa: después de un día sin refrigeración todavía no se había deshecho. Tal característica fue valorizada por las amas de casa como muy importante en las condiciones de Pueblo Nuevo.

\section{Prácticas de selección}

Se tomaron datos de: emergencia, floración y resistencia al MD. En dos momentos fueron a evaluar los 45 agricultores adicionales (después de la floración y en la cosecha). Sin embargo, el momento más importante para la selección fue en la cosecha. Se cosecharon únicamente plantas con poca incidencia de MD (estaban marcadas o las plantas con MD fueron eliminadas). Con base en el rendimiento y el color del grano se seleccionaron las familias y dentro de las familias las plantas individuales (postrera 2000, primera y postrera 2001). Resultó difícil para los agricultores limitarse a los números que se había acordado en las reuniones, y con base en los consejos del fitomejorador. Entonces, fue una práctica normal que antes de la siembra se efectuara una segunda selección, para eliminar algunas familias o plantas, reduciendo el número de materiales para la siembra. Los rendimientos se determinaron en los ensayos de comparación de 2002 (campaña de riego) y en los ciclos siguientes. Los datos del Cuadro 2 indican que todos los materiales seleccionados produjeron igual o mejor que el testigo INTA Masatepe.

Los agricultores-fitomejoradores tenían suficiente experiencia para comprobar que los materiales seleccionados por ellos tenían un comportamiento superior a las variedades de frijol disponibles en este momento. Además, tenían como referencia sus campos y los de sus vecinos sembrados con estas variedades. Esto explica por que no se sentía la necesidad de ensayos de comprobación hasta fines de 2002 en el cual se comparaba los materiales de los cinco agricultores-fitomejoradores con un testigo. Otro elemento fue la necesidad de incrementar el volumen de semilla para tener suficiente grano para ensayos de prueba.

\section{Comportamiento de los materiales seleccionados}

Para comparar los cinco mejores materiales de los cinco agricultores-fitomejoradores y conocer su comportamiento en diferentes ambientes, se sembraron varios ensayos. En una siembra de riego en 2001 y primera de 2002 se compararon los materiales en campo de los cinco agricultores bajo manejo del agricultor (Cuadro 2). En cuatro casos salió con mayor 
Cuadro 2. Rendimiento ( $\mathrm{kg} / \mathrm{ha}$ ) de las cinco mejores líneas de frijol seleccionadas por los cinco agricultores-fitomejoradores en parcelas de evaluación (sin repeticiones) sembradas en sus fincas. Nicaragua, 2005.

\begin{tabular}{lrccccc}
\hline & \multicolumn{5}{c}{ Origen de material (agricultor) } \\
\cline { 2 - 7 } $\begin{array}{l}\text { Agricultor (y localidad) } \\
\text { de siembra de ensayos }\end{array}$ & Juan García & Jose M. González & Pedro Gómez Santos L. Merlo & Jairo Videa & Testigo $^{2}$ \\
\hline García (Santa Rosa) & 2.005 & 1.551 & $2.717 *$ & 2.069 & 2.127 & 1.875 \\
Gómez (La Lima) & 969 & 839 & $1.948 *$ & 1.098 & 1.164 & 1.551 \\
González (Paso Hondo) & 969 & $2.522 *$ & 2.134 & 2.134 & 2.263 & 1.616 \\
Merlo (El Rosario) & 1.035 & 1.016 & 1.180 & $1.722 *$ & 1.275 & 1.057 \\
Videa ( Río Abajo) & 2.328 & 1.616 & 1.357 & 1.482 & $2.522 *$ & 2.269 \\
\hline
\end{tabular}

1 Para detalles de agricultores y sus campos, véase Cuadro 1.

2 En la siembra en el campo de Santos Luis Merlo se usó como testigo DOR 364, en las otras siembras INTA-Masatepe.

* El material con mayor rendimiento en el ensayo de cada de los cinco agricultores.

rendimiento el material seleccionado por el agricultor mismo. Vale mencionar que se le había proporcionado el material bajo código y que entonces los agricultores no estaban informados de cual material fue el suyo.

En la postrera de 2002 se sembraron las cinco líneas en 26 diferentes ensayos. Los ensayos fueran establecidos en los diferentes ambientes agro-ecológicos de Pueblo Nuevo y Condega, representando las diferentes condiciones presentes en la localidad. Con base a los análisis estadísticos (Molina et al. 20036) las líneas de José M. González y Juan García resultaron las mejores por su rendimiento y adaptación. En un análisis de los rendimientos de los 14 ensayos que tenían un índice ambiental mayor que el promedio de los 26 ensayos, INTA-Masatepe salió como mejor, aunque su rendimiento $(852 \mathrm{~kg} / \mathrm{ha})$ no fue significativamente mayor que la del material de José M. González ( $850 \mathrm{~kg} / \mathrm{ha})$ (Molina et al. 20036). En el análisis de rendimiento sobre los 12 ensayos con índice ambiental menor que el promedio de los 26 ensayos, salieron los materiales de José M. González y de Juan García con mayor rendimiento (470 y $457 \mathrm{~kg} / \mathrm{ha}$, respectivamente) que el testigo INTA-Masatepe (437 kg/ha) (Molina et al. 20036).

En la primera de 2003 se sembraron 22 ensayos con semilla de las dos líneas superiores de la campaña

\footnotetext{
6 MOLINA, J.C.; ALFARO, N.; HERRERA, R. 2003. Validación de líneas de frijol de grano rojo en Nicaragua (Comunicación personal). Instituto Nicaragüense de Tecnología Agropecuaria. Managua, Nicaragua. Correo electrónico: juliomolinac@hotmail.com
}

anterior; 19 parcelas fueron cosechados (Molina et al. $2003^{6}$ ). En esta serie de ensayos el rendimiento del material de José M. González superó al testigo INTAMasatepe (Cuadro 3), una diferencia que fue significativa (Molina et al. 20036).

Sobre los 41 ensayos, el material de José M. González salió con un rendimiento significativamente mayor en rendimiento que INTA-Masatepe y que el material de Juan García (Molina et al. 20036). Los coeficientes de regresión $\left(\mathrm{R}^{2}\right)$ del material de José M. González $(0,93)$ y Juan García $(0,90)$ superaron ligeramente el de INTAMasatepe $(0,87)$, expresando una ligera superioridad en estabilidad a través de las localidades.

En la cosecha de la primera de 2002 también se hizo una prueba culinaria con los agricultores y sus cónyugues. En esta prueba sobresalió también el material de José M. González como el mejor. La característica de mayor valor fue la consistencia en la sopa después de haber sido guardada un día sin refrigeración y recalentada. El color y tamaño del grano de esta línea, tanto como tiempo necesario para cocinar fueron también considerados superiores al testigo INTA-Masatepe.

El grupo de agricultores estuvo de acuerdo en enfocarse en estos dos materiales que fueron nombrados Pueblo Nuevo JM 12-7 y Santa Elena. Se tomaron iniciativas para tener las variedades registradas en el Ministerio de Agricultura y Forestales (MAGFOR). Con los datos que tomó el fitomejorador de los ensayos de comprobación en 2002 (26) y 2003 (22) se cumplió 
Cuadro 3. Rendimiento promedio (kg/ha) de las cinco mejores líneas de frijol seleccionadas por los cinco agricultores-fitomejoradores en ensayos sembrados en Pueblo Nuevo (26) y Condega (22) en la postrera 2002 y primera 2003, respectivamente. Nicaragua. (Molina 2003, Comunicación personal7).

\begin{tabular}{|c|c|c|c|c|c|c|}
\hline & \multicolumn{6}{|c|}{ Origen del material (agricultor-fitomejorador) } \\
\hline & Juan García & Jose M. González & Pedro Gómez & Santos L. Merlo & Jairo Videa & $\begin{array}{c}\text { INTA - } \\
\text { Masatepe }\end{array}$ \\
\hline $\begin{array}{l}\text { Promedio de } 14 \text { ensayos } \\
\text { en ambientes buenos *, } \\
2003\end{array}$ & 610 & 675 & 601 & 549 & 619 & 660 \\
\hline $\begin{array}{l}\text { Promedio de } 12 \text { ensayos } \\
\text { en ambientes pobres *, } \\
2003\end{array}$ & 457 & 470 & 406 & 353 & 334 & 437 \\
\hline $\begin{array}{l}\text { Promedio de } 22 \text { ensayos, } \\
2004\end{array}$ & 538 & 514 & - & - & - & 526 \\
\hline
\end{tabular}

* La media general de índice ambiental fue $618 \mathrm{~kg} / \mathrm{ha}$.

con los requisitos de una inscripción. También se produjeron 40 quintales de semilla y se formó una cooperativa para disponer de un ente jurídico al cual pertenece la variedad. Se lanzaron las dos variedades en octubre 2004 y posteriormente la cooperativa produjo y vendió 40 quintales de semilla de calidad 'artesanal'. A finales del 2005, no se había logrado registrar las variedades todavía. La ley de semillas vigente en Nicaragua no puede incluir como fitomejorador, a una cooperativa que no está así inscrita, en cumplimiento de una serie de requisitos técnicos.

\section{DISCUSIÓN}

Las experiencias claramente demuestran que los agricultores tienen condiciones que permiten el desarrollo de variedades a partir de materiales segregantes. Fue demostrado antes en un ensayo en Colombia (Kornegay 1995), con la diferencia que en el caso aquí presentado las dos variedades que salieron fueron superiores a la variedad comercial de más uso en ese momento, el INTA-Masatepe. Su superioridad en primer lugar fue por su color, calidad culinaria y, según los agricultores, en su resistencia al Mosaico Dorado y tolerancia a sequía, resultando en buenos rendimientos. Estas son características que no se distinguen con facilidad en los ensayos de comprobación con base en el rendimiento, pero que son de mucha importancia para los pequeños agricultores: ya que les permite producir relativamente con pocos insumos (químicos, riego) y vender la cosecha a buen precio y por su calidad para consumo. En términos de rendimiento, el Pueblo Nuevo JM 12-7 y Santa Elena mostraron su buen comportamiento, más claro en los ambientes mas pobres (índice ambiental menor que el promedio de $618 \mathrm{~kg} / \mathrm{ha}$ ). Eso puede dar apoyo al argumento que sostiene que FP tiene mayor importancia en ambientes marginales. Sin embargo, se necesita tomar en cuenta que también INTA-Mazatepe rindió bien en estos ambientes pobres y que el coeficiente de regresión (medida para la estabilidad de rendimiento) apenas fue menor que de JM 12-7. Esto confirma que la preferencia de los agricultores para los materiales seleccionados por los agricultores, se basa en otras características, además del rendimiento.

El hecho que JM 12-7 posee el coeficiente mayor de los materiales evaluados (indicando que era el material con mayor estabilidad de rendimiento), no apoya la hipótesis que dice que el FP resulta para materiales de adaptación específica. Por otro lado, la observación que indica que el material más productivo en los campos de los agricultores fue en cuatro de los cinco casos el material del mismo agricultor, es algo que indica una adaptación específica al ambiente en lo cual el material fue seleccionado. La baja estabilidad del rendimiento de Santa Elena se debe a que fue una variedad seleccionada a $1.000 \mathrm{msnm}$ y adaptada al clima en estas altitudes. 


\section{Aprendizajes acerca de metodología de selección}

En reflexión sobre las experiencias, los agricultores-fitomejoradores llegaron a la conclusión de que había tres puntos importantes:

- La selección entre materiales segregantes de frijol en campo de agricultores y manejados por ellos fue factible

- Tener cinco iniciativas individuales paralelas con una comparación al final no tenía mucho sentido. Se podría ganar eficiencia en comparar materiales en fases más tempranas o reducir el número de agricultores-fitomejoradores que llevaran parcelas de evaluación.

- Basar las pruebas culinarias sólo en las líneas promisorias para uso comercial significó mucho riesgo. Que la variedad Pueblo Nuevo JM 12.7 saliera con buenas características culinarias en realidad fue un asunto de suerte: no habían considerado las características culinarias hasta el final del proceso a pesar de que es un criterio muy importante para las esposas de agricultores.

Mejorar la metodología en estos puntos sería más fácil si se pudiera disponer de mayores volúmenes de semilla en la primera fase del proceso. Sin embargo, en fitomejoramiento, tener poca semilla en generaciones tempranas es un fenómeno inevitable sin perder eficiencia en el proceso.

\section{La participación}

La experiencia del proyecto da espacio a algunas pautas relacionadas con la participación, relevante para el Fitomejoramiento Participativo como una estrategia para desarrollar variedades adaptadas a condiciones de pequeños agricultores.

\section{Las condiciones específicas}

El proyecto se inició cuando en las comunidades no se disponían de buenas variedades de frijol con resistencia. En la región de Mesoamérica la incidencia de MD se incrementó fuertemente en las últimas dos décadas como consecuencia de la intensificación de la producción de tabaco y tomate. Las variedades locales tienen poca resistencia; la variedad DOR 364 tenía algo de resistencia pero el color rojo oscuro de sus semillas no permitió obtener un buen precio en la comercialización. Estos fueron factores de mucha importancia en la motivación de los agricultores de participar en un proyecto, que no tenía el éxito garantizado y lo cual requería un compromiso serio a un plazo mediano- largo. Al terminar el proceso, en 2004, se disponía también de INTA-Masatepe, una variedad con características más adaptadas. Sin embargo, en comparación con Pueblo Nuevo JM 12-7 y Santa Elena, los agricultores la consideran inferior en rendimiento, valor comercial y calidad culinaria. Los agricultores también manifiestan que valorizan Pueblo Nuevo JM 12-7 por su calidad de grano, mientras Santa Elena es valorada específicamente en las alturas donde se produce una parte significativa de la producción frijolera de Nicaragua (arriba de los $850 \mathrm{msnm}$ ). En este momento, los agricultores mantienen un nivel alto de interés en participar en proyectos de FP. Sin embargo, se puede preguntarse si es posible mantener el nivel de éxito en términos de sacar nuevas variedades y satisfacer las expectativas de los agricultores relacionado con la venta de semilla.

El fitomejorador fue el especialista que inicialmente tenía el peso más importante en las decisiones tomadas por el grupo en las reuniones. Fue un peso no explícitamente buscado, pero necesario debido a la falta de conocimiento de parte de los agricultores y técnicos acerca de la fitogenética. Con explicaciones (talleres de aprendizaje-planificación) y las experiencias mismas, los agricultores y técnicos incrementaron su confianza y criterios de decisiones. Así fue que un agricultor, a pesar de que se había acordado no aplicar riego porque se buscaba tolerancia a la sequía, tomó la decisión de regar. Entonces, se ha podido notar que, con el desarrollo del proyecto, la relación entre agricultores y fitomejorador se evaluó de una de alumnos-profesor a otra en la cual todos fueron alumnos, aprendiendo de las experiencias. El fitomejorador manifestó que al final, después de trabajar varios años en frijol, realmente 'conoce' qué color de frijol buscan los agricultores. Los agricultores ahora entienden de donde se originan las variedades nuevas. Discuten entre ellos sobre ventajas y desventajas de trabajar con materiales segregantes vs líneas avanzadas. Unos dicen que prefieren que les den líneas avanzadas porque es menos trabajo. Otros, sin embargo, prefieren materiales segregantes porque les da más diversidad y entonces un mayor "chance" que pueden seleccionar la 
variedad a sus gustos. Algunos están con curiosidad de aprender como cruzar frijoles porque así no estarán dependiendo de otras organizaciones para conseguirse materiales de selección.

\section{Uso de diversidad en campo de agricultores}

Un argumento que se usa en apoyo de FP es que sería una estrategia de fitomejoramiento que llegara hacer disponible mucha más diversidad genética al agricultor. La diversidad genética en términos generales favorecería la sostenibilidad del sistema agro-ecológico y daría oportunidades de mantener germoplasma de alto valor, no solamente para el agricultor, pero también para la humanidad. En el caso del proyecto aquí documentado se podría decir que FP ha contribuido al uso de mayor diversidad genética al campo del agricultor en Pueblo Nuevo y Condega. Se seleccionaron dos materiales que los agricultores habían avanzado hacia variedades supuestamente con una homogeneidad genética similar a cualquier variedad mejorada generado por medio de programas convencionales. Estos dos materiales se encuentran sembrados en Pueblo Nuevo, Condega y otras zonas donde se están difundiendo, en adicción al portafolio ya existente. Encima de esto, los agricultores en el transcurso del proyecto, desde que se arrancó, han intercambiado y regalado semillas a amigos, vecinos y familiares. Un material de uno de los cinco agricultores es conocido por su barrio como "Luisito", el nombre del agricultor. El material "Luisito" no salió como superior en los ensayos de prueba, pero los agricultores lo valorizan por su calidad culinaria y resistencia a la sequía. Para tener información más exacta acerca del impacto de FP sobre el uso de diversidad genética en los campos se necesitará un estudio que realice un inventario y que siguiera como se cambia el portafolio de variedades que los agricultores siembran, y como se difunde el material tanto dentro como fuera de las localidades. Finalmente, merece anotar que en este caso, se partieron de materiales segregantes de tres cruces triples lo cual no incluía variedades locales de la zona. Ahora los agricultores están evaluando y seleccionando materiales que son cruces de variedades locales que ellos indicaron como valiosos con materiales que confieren resistencia a sequía y MD. Estos cruces, hechos en la Escuela Agrícola Panamericana El Zamorano, no estaban disponibles al inicio del proyecto. Tales materiales pueden contribuir significativamente a la conservación in situ de la diversidad genética que tiene un valor a nivel global.

\section{CONCLUSIÓN}

La experiencia demuestra que se ha logrado desarrollar por medio de una investigación acción una metodología de FP con lo cual se pueden desarrollar variedades superiores en campo de agricultores y con conocimientos de ellos. La experiencia fue valorada positivamente por todos los participantes, incluyendo las agencias donantes. Eso da lugar a dos retos: (1) mejorar esta metodología y (2) buscar la manera en lo cual FP podría complementar o ser integrado al fitomejoramiento convencional del INTA o instituciones internacionales. ¿Cuáles serán los costos y cómo se cubrirían estos? Los agricultores en Pueblo Nuevo y Condega ahora tienen en su campo diferentes ensayos de evaluación, incluyendo ensayos del CIAT. Los agricultores tienen motivación y capacidad y son entonces colaboradores atractivos para los fitomejoradores. En este proyecto piloto los agricultores no fueron pagados, solamente costos extra-ordinarios como gasolina para la bomba de riego, fueron pagados por el proyecto, es decir por una agencia donante. Entonces, en ninguna manera este proyecto ha afectado los fondos disponibles para los programas convencionales. $\mathrm{La}$ motivación de los agricultores se origina en el orgullo, el prestigio y el aprendizaje que sacaron de la experiencia. Pero ¿cómo se mantendría esta motivación en una colaboración con fitomejoradores en un plazo más largo? En este sentido es importante preguntarse ¿qué tan fácil será desarrollar materiales que superan a Pueblo Nuevo JM 12-7 y Santa Elena? Y, ¿qué posibilidades habrá de recuperar los costos de lo cual ellos ahora se encargan, es decir, costos de terreno, riego y tiempo? El JM y Santa Elena no parecen tener limitaciones en su difusión que se podría atribuir a adaptación específica a las condiciones de Pueblo Nuevo y Condega. Pero, con fitomejoradores del INTA también trabajando con mejores materiales y criterios, y con otras comunidades también entrando en FP, ¿dónde estaría la fuente o cuál sería el papel de los agricultoresfitomejoradores en Pueblo Nuevo y Condega?. Esto sugiere un re-pensar de FP en términos de descentralización y conexión con el sistema de producción y 
difusión de semilla. El escalamiento y la institucionalización de FP es un punto tal vez más complejo todavía que el FP que implementaron en este proyecto piloto. Es imposible pensar y diseñar todos los aspectos y respuestas en la oficina: requiere una estrategia en la cual 'se desarrolla haciendo e implementando', con una colaboración entre un rango amplio de actores.

\section{AGRADECIMIENTO}

Los autores quieren agradecer a todos los actores involucrados. En especial se agradece a los 45 agricultores que participaron en reuniones, evaluaciones y otros eventos, contribuyendo con su tiempo, conocimiento y criterios. A Pedro Gómez y Jairo Videa, agricultores y fitomejoradores, socios de la Cooperativa de Semillas de Pueblo Nuevo (COSENUP R.L.), quienes participaron como experimentadores y productores de semillas. También se agradece Norman Alfaro y Javier Pasquier de CIPRES por su coordinación y facilitación del proyecto. A NORAD y Fondo de Desarrollo se agradece por su provisión de recursos financieros. A los editores de la revista se les agradece por sus correcciones, sus sugerencias y cuidadoso trato del texto.

\section{LITERATURA CITADA}

ALMEKINDERS, C.; ELINGS, A. 2001. Collaboration of farmers and breeders: participatory crop improvement in perspective. In: Elings, A.; Almekinders, C.; Stam, P. eds. Breeding for low-input conditions, and consequences for Participatory Plant Breeding. Euphytica 122 (3): 425-438.

ALMEKINDERS, C.; MOLINA, J.; ALFARO, N.; HERRERA, R. 2002. Fitomejoramiento participativo en Nicaragua ya es un éxito para los involucrados. Científicos y agricultores logrando variedades mejores. Memoria de la Asamblea Annual del Comité Mesoaméricano, Managua, Nicaragua, 28-30 de Mayo de 2001. Fondo de Desarrollo De Noruega/CIPRES, Managua. p 87-93. (en línea) Consultado 6 feb. 2006. Disponible en: www.programa-fpma.org.ni

BERG, T. 1996. Devolution of plant breeding. In: Sperling, L.; Loevinsohn, M. eds. Enhancing and maintaining genetic resources on farm. New Delhi, India. p. 116123.

CBD. 1992. Convention on Biological Diversity. UNEP. (en línea). Disponible en : http: //www.biodiv.org

HARDON, J. 1995. Participatory plant breeding. The outcome of a workshop on participatory plant breeding, 2629 July 1995. Plant Genetic Resources No. 3, October 1995. IPGRI, Rome.

KORNEGAY, J.; ALONSO, J.; ASHBY, J. 1996. Farmer selections within segregating populations of common bean in Colombia. In: Eyzaguirre, P.; Iwanaga, M. eds. Wageningen, The Netherlands. Participatory Plant Breeding. p. 151-159.

SPERLING, L.; ASHBY, J.A.; SMITH, M.E.; WELTZIEN, E.; MCGUIRE, S. 2001. A framework for analyzing participatory plant breeding approaches and results. Euphytica 122 (3) 439-450. 